\title{
A Novel Approach to Laparoscopic Colonoscopic Polypectomies
}

\author{
James JL Mateka ${ }^{1 *}$, Michael M Haniff ${ }^{1}$ and Donald M Davis ${ }^{2}$ \\ ${ }^{1}$ Rush University Medical Center, USA \\ ${ }^{2}$ University of South Florida, USA
}

Submission: March 14, 2017; Published: March 20, 2017

*Corresponding author: James JL Mateka, Rush University Medical Center, USA, Email: jamesmateka@gmail.com

\begin{abstract}
Introduction: Many benign colorectal polyps cannot be positioned correctly or reached by the endoscope for polypectomy. Accordingly, patients undergo surgical resection of involved bowel. Due to this fact, we have attempted to develop minimally invasive techniques in porcine models to assist with endoscopic polypectomies. The MiniLap is a novel device that deploys a grasping mechanism through a needle-tipped shaft. We used this device for dissection, exposure, retraction, as well as transcolonic insertion to assist in this endeavor.
\end{abstract}

Methods: Under IACUC approval, using a supine porcine model, two $12 \mathrm{~mm}$ ports are placed (periumbilical and right lower quadrant) for a camera and instrumentation. Minilaps are inserted to assist with exposing, retracting, and dissecting. Once the area of polypectomy was exposed, the MiniLap was inserted (using the needle tip) transcolonically to present the polyp to the endoscope. After polypectomy, the colotomy was closed with a purse string and instruments were removed.

Results: We were able to simulate endoscopic polypectomies with transcolonic assistance from the MiniLap for polyps that would normally be inaccessible. Intracolonically, the MiniLap can be placed through a loop cautery and used to grasp the polyp. The loop then slides over the device to the base of the polyp. Another technique uses the MiniLap to grasp proximal mucosa and display the polyp; making it accessible to the endoscope.

Conclusion: In the porcine model, the MiniLap can be used to assist endoscopic removal of difficult to reach polyps. This will hopefully translate into decreased bowel resections, morbidity and hospital stay in human subjects.

Keywords: Benign polyps; Polypectomy

\section{Background}

William Wolff and Hiromi Shinya were among the first to develop and implement endoscopic polypectomies for treatment of colorectal polyps [1]. Several advances have been made throughout the past decade to make this outpatient procedure a safe form of treatment. Endoscopic polypectomy remains the first tier of treatment for colonic polyps [2]. The alternative tier of treatment is surgical resection. Surgical resection carries the burden of hospitalization cost which may be avoided if colonic polyps can be removed endoscopically [3]. Although considered a safe treatment for these polyps, endoscopic polypectomy does increase the potential risk for hemorrhage and perforation of the bowel [3]. Previous studies of endoscopic polypectomies have revealed complication rates ranging from 1 to $9 \%$ [3].

Colonic polyps found during colonoscopy are typically biopsied or removed using cold or hot snares. At times it is very difficult to remove these polyps because they may be large or flat, risking bowel perforation in the process. Additionally, access to the polyp may be difficult due to adhesion from previous surgeries or redundancy of the colon and makes removal of the polyp also difficult. Traditionally, these patients would have to undergo a bowel resection to remove the polyp consequentially with many associated complications. Over the last decade, many cases for colon polyps not amendable to colonoscopic removal have been referred for surgical removal. This novel approach deals with the issue head on of unnecessary bowel resection for benign polyps allowing colonoscopies to be performed in the operating room [4].

We can be more aggressive in our polypectomies due to the fact that if perforation occurs we can immediately repair it. If warranted, laparoscopic assistance is used for enhanced colonic visualization, mobilization of the bowel and, if required, takedown of adhesions. This procedure allows access to polyps that we not previously accessible with traditional colonoscopy. This 
approach also allows the laparoscopic repair of any colotomy made and buttresses any serosal tear preventing unnecessary bowel resections. The MiniLap device can be used to aid in mobilization of the colon without having to add formal ports for traditional graspers (as we currently do), as well as aid in handling the colon itself. The MiniLap device was inserted through the colon wall into the lumen of the colon to grasp the polyp itself.

By doing this we could retract the polyp towards the colonoscope to add in colonoscopic removal. If Colonoscopic removal is not feasible, we used the MiniLap to precisely locate and grasp the polyp for removal by simple colotomy with simple laparoscopic closure, thus also adverting an open bowel resection. A retrospective chart review of our institution revealed 106 patients with a diagnosis of benign polyps by colonoscopy underwent repeat intraoperative colonoscopies with repeat intraoperative colonoscopic polypectomies with laparoscopic assistance between March 2001 and September 2009 [5]. The median age was 65 with $56 \%$ male patients. of these patients, 74 (70\%) were successfully treated with colonoscopic polypectomy, 10 of which utilized laparoscopy for mobilization and positioning of the colon.

Of the 32 operative patients (30\%), there were 17 colectomies, 7 cecectomies, 5 transanal excisions, 2 low anterior resections, and one colotomy with mass excision. These patients underwent formal resections due to size, inaccessibility or appearance suspicious for malignancy. Upon analysis, we found a significant difference in the length of hospital stay between these two groups $(p<0.01)$. The mean stay for the non-operative group was less than one day (median 0), while the operative group had a mean stay of 5 days (median 4). Complications included 1 perforation during colonoscopy (recognized at the time of operation), 1 patient with continued rectal bleeding after colonoscopy (self-resolving), 2 carcinomas originally diagnosed as benign, 1 anastomotic leak, and 1 perioperative surgical site infection.

Repeating the colonoscopy intraoperatively with laparoscopic assistance appeared to have an overwhelming benefit. Thus, we anticipate an increase in the number of successful treatments through MiniLap. In addition, with the use of the MiniLap we may be able to treat many of the other patients with simple colotomy, polypectomy and simple laparoscopic closure, avoiding an open formal bowel resection and its associated complications and prolonged hospital stay. This project was completed with a total of five milestones. Each milestone was intended to further develop the surgeons' skill and familiarity with the device. Summary descriptions of each milestone can be found in (Table 1). This purpose of this project was to demonstrate the role of the MiniLap device in laparoscopic assisted colonoscopic polypectomies. We hoped to demonstrate how the device may make more patients eligible for colonoscopic polypectomies with improved cosmesis and decreased number of formal laparoscopic ports [6,7].

Table 1: In-depth Milestone Description.

\begin{tabular}{|c|c|}
\hline Milestone & Description \\
\hline & $\begin{array}{c}\text { Familiarization with equipment and its use } \\
\text { intracorporeally and }\end{array}$ \\
intralumenally. $\begin{array}{c}\text { During this initial lab, we will } \\
\text { familiarize }\end{array}$ \\
ourselves with the MiniLap Device. As a team we will \\
discuss \\
and experiment with best locations for insertion as wel \\
as exact \\
methods of use for the MiniLap device.
\end{tabular}

Using the MiniLap in polypectomy models

During this lab we will attempt to perform mobilization of the

colon with the use of the MiniLap for retraction. We will then

use the MiniLap for simulation of transcolonic intraluminal

2

retraction of a mucosal segment with colonoscopic removal,

mimicking colonoscopic polypectomy. The lab will be

concluded with an attempt at removing a mucosal segment

precisely with the assistance of the MiniLap to grasp the

mucosal segment while a colotomy is made and then repaired

laparoscopically, mimicking a laparoscopic assisted polypectomy with colotomy closure.

Refinement and adjustments to the polypectomy models. During

this lab we will complete any of the simulations that we were not

able to complete during the previous session. In addition, we will

modify and attempt to approve the efficiency of the models we

first performed during the previous session.

\begin{tabular}{|c|c|}
\hline & $\begin{array}{l}\text { Recording of Polypectomy Model. During this session } \\
\text { we will }\end{array}$ \\
\hline 4 & $\begin{array}{l}\text { continue to modify the procedures as needed. We will } \\
\text { also record }\end{array}$ \\
\hline & the procedures for further study and demonstration. \\
\hline
\end{tabular}




\begin{tabular}{|c|c|}
\hline 5 & $\begin{array}{l}\text { Final Modification to Pig Model. During this session, we } \\
\text { will } \\
\text { make any final adjustments to the procedures after } \\
\text { reviewing the } \\
\text { previous session's videos. We will then record any } \\
\text { changes to } \\
\text { the procedures in preparation for use in future } \\
\text { demonstrations } \\
\text { and possible use in humans. We will then edit the video } \\
\text { and } \\
\text { prepare for possible presentation. }\end{array}$ \\
\hline
\end{tabular}

\section{Materials and Methods}

Under Institutional Animal Care \& Use Committee (IACUC) approval this project was carried out in a swine (pig) model. Three days before the planned operation the pig began a bowel prep to provide as much catharsis of fecal material as possible before the proposed procedure. On the day of the procedure, the pig was placed under general anesthesia by trained animal lab staff. The pig was placed in the supine position. A colonoscope was placed per rectum. Laparoscopic ports were inserted in positions deemed appropriate at the time of operation. The MiniLap devices were inserted transabdominally and used for mobilization and dissection of the colon. We also planned to insert the MiniLap devices through the colon wall in order to grasp the lumen of the colon and mimic assistance for a colonoscopic polypectomy. This process was visualized with the colonoscope. We then explored the use of the MiniLap in grasping mucosa through the colon and excising this segment with simple colotomy and laparoscopic closure, with the mucosal segment once again representing a polyp. In this way we hoped to determine if the MiniLap device can feasible be used in humans to prevent formal bowel resections for benign polyps. After the procedure the pig were euthanized in an IACUC approved manner.

\section{Results}

We developed the proper techniques for safely inserting the device into the pig. We discovered that a separate stab incision did not need to be made in order to safely enter the abdominal cavity. We found that separate MiniLaps inserted into the left and right upper quadrants allowed for good triangulation (along with an umbilical camera port) for dissection. We were able to insert the MiniLap through the colon and close the colotomy with a purse string closure during this session as well. During session two, we were able to complete a simulation of a transabdominal, transcolonic polypectomies. We found a novel use for the MiniLap as well. We used multiple MiniLaps inserted into the abdominal cavity as self-retaining retractors after grabbing viscera (such as bladder).

We also found that placing a U-stitch around the area where the colotomy is going to be made provides for additional retraction and allows the colotomy to be closed at the same time that the MiniLap is being removed from the colon (the suture is tightened as the device is removed), minimizing any fecal contamination. We also attempted to close the colotomy with clips/staples, but found this to be more difficult and less successful. Finally, we attempted to find pitfalls with using the MiniLap. We were able (although with some difficulty) to injure the bowel by grabbing too hard with the MiniLap and trapping bowel inside the needle.

We were able to create serosal injuries, but we were not able to make full thickness injuries. We also tried to injure blood vessels within the abdominal wall, but found that the vessels seemed to "roll" out of the way. Through completing session three, we attempted to refine and explore other techniques for a transabdominal, transcolonic polypectomies. In order to only use an umbilical port (similar to single incision surgery) and the MiniLaps, we attempted to place our U stitch and perform a colotomy closure using a straight needle placed through the abdominal wall and tie the knots intracorporeally, This proved very difficult in the narrow porcine abdomen and we caused bowel injury before successfully closing the colotomy. We then changed our plan and placed a port near our umbilical port to simulate a single incision port. We were able to close the colotomy effectively.

During session four, we were able to complete the transabdominal, transcolonic polypectomy through a single incision port. We did this using a $5 \mathrm{~mm}$, rigid, 30-degree camera. We found that the procedure was difficult and the colotomy closure was less precise. The colotomy closures did encompass more bowel and appeared to stricture the bowel somewhat externally, but intraluminally (as viewed through the colonoscope) there was minimal narrowing and the scope easily passed through. Although we demonstrated the procedure was feasible through a single incision with assistance from the MiniLap, more refinements were needed (a flexible tipped scope would also be of tremendous help in this procedure).

During the last session, session five, we attempted to use the techniques that we had developed to truly simulate a transabdominal, transcolonic polypectomy. We decided to use a two port technique with an umbilical port and a right lower quadrant port. We used a MiniLap in the low midline to be used as a self-retaining retractor for the bladder. We used right upper and left upper MiniLaps for retraction and exposure, and a infraumbilical MiniLap in the midline for the polypectomy. The exposure was optimal. A U stitch was placed at the area of colotomy. The MiniLap was placed through the colon wall into the lumen. A simulated polypectomy was performed lifting mucosa from behind a fold and presenting it to the colonoscope. The colotomy was closed with the U stitch that had been previously placed. Ensuring the closure was air tight, the instruments were then removed. We wanted this simulation to go as flawlessly as 
possible so we performed this with optimal exposure and did not attempt a single incision technique, although we had previously proved that this was feasible.

\section{Discussion}

Each individual milestone set forth at the beginning of the project was successfully completed. During session one, we first inserted the device by making a "puncture" with a scalpel through the skin, followed by inserting the needle-point of the MiniLap through the subcutaneous tissue and fascia into the abdominal cavity without difficulty. However, we also found that we were easily capable of inserting the device without creating the "puncture" with the scalpel. We used the needle tip of the MiniLap to pierce the skin/subcutaneous tissue/fascia. We held the shaft of the device $2-4 \mathrm{~cm}$ proximal to the needle tip to ensure that the device did not penetrate too deep into the abdominal cavity and injure viscera.

The cosmesis through this technique was much improved. In addition, we attempted to injure superficial blood vessels within the abdominal wall (this is sometimes done inadvertently when placing laparoscopic ports). This was done easily when using the scalpel, however, when using the MiniLap, this was surprisingly difficult. The vessels seem to "roll" and actually puncturing those (causing bleeding) took effort and multiple attempts. Location of insertion was determined after examining the abdomen through a laparoscopic camera in an umbilical port, we decided to place our initial MiniLaps in the right and left upper quadrants. These sites allowed us to retract bowel effectively. An additional MiniLap was eventually placed in the lower quadrant at midline for transcolonic insertion. This location would vary depending on the location of the polyp to be removed. It appeared that these locations were appropriate, although we may try to complete the procedure without either the left or right upper quadrant sites in the future.

The device was then inserted into the bowel. Transcolonic insertion initially proved to be difficult. Simply using insufflation from the endoscope as counter resistance and inserting the MiniLap through the colon wall caused unintended injury. Multiple attempts were made unsuccessfully as the MiniLap would slide from its intended insertion site. This would cause serosal tearing. This problem was solved by grasping the colon just proximal to its intended insertion site with another MiniLap. This provided enough counter resistance for the other MiniLap to be inserted into its intended site. Although we effectively inserted the MiniLap transcolonically, further technique improvements are still needed.

Finally, we closed the colotomy using an endostitch device. We created a purse string closure with a silk stitch. This very effectively closed the colotomy. The closure was air tight (maintained insufflation) and had very minimal narrowing as seen from the endoscope. Although effective, we would like to refine the closure in future sessions. Session one indicated, the MiniLap device can be inserted safely with direct insertion into the abdominal cavity. A device inserted into the right and left upper quadrant helps assist in dissection. The MiniLap can be inserted transcolonically for assistance in endoscopic polypectomies. The colotomy made can effectively be closed with a purse string closure.

As we moved forward to session two, mobilizing, dissecting, retracting, and exposing the colon was attempted. In order to expose the colon, small and large bowel must be moved. We found this could be done either by sweeping the bowel with the grasper closed or using the grasper with gentle closure and placing the bowel in the appropriate quadrants. This session's pig had a persistent urachus which had to be taken done with electrocautery dissection and gentle retraction. This was done easily with the MiniLaps and a single incision umbilical port (the camera and electrocautery were placed through the same port).

The MiniLap was surprisingly very effective as a selfretaining retractor. The abnormally large bladder was grasped with the MiniLap and the extracorporeal disk of the MiniLap was placed flush with the abdominal wall providing retraction. A similar tactic was used with bowel in the right upper quadrant. The area for planned polypectomy was completely exposed at this point. We then used both a preplaced purse string suture and extra MiniLap grasping proximal to provide counter resistance. The MiniLap's needle point was easily introduced into the colon under endoscopic visualization, at this point we grasped mucosa. We used snare cautery to remove mucosal segments. We found this could be done in one of two ways. The first was to grab nearby mucosa and present the "polyp" to the endoscope.

The second method involved threading the MiniLap through the loop of the snare, then grabbing the tip of the "polyp", sliding the snare over the MiniLap to the base of the "polyp". At this point during the project we developed potential improvements which should be explored. We found that placing a U-stitch around the area where the colotomy is going to be made provides for additional retraction and allows the colotomy to be closed at the same time that the MiniLap is being removed from the colon (the suture is tightened as the device is removed), minimizing any fecal contamination. We attempted colotomy closure with staples with one successful attempt and one unsuccessful attempt. It was clear that the U-stitch method was both easier and more effective. There are several potential dangers that were observed during this session. We intentionally attempted to injure bowel with the MiniLap device, which was not easy, but possible.

By taking large bites of bowel/viscera and closing the device, we could cause significant serosal tearing (mainly by taken tissue into the needle-tipped shaft), although we were not able to penetrate the bowel/viscera. We also found that we could grasp part of the mesentery containing the blood supply and cause ischemia. We originally attempted to perform the 
suturing through a single incision port with the camera, limiting the procedure to one traditional incision in the umbilicus. This was very difficult and resulted in the suture sawing through the bowel wall and causing a large colotomy. The procedure was then completed after a new port was inserted into the left lower quadrant for the endostitch. We would like to refine our technique and attempt the procedure as a single incision with MiniLap assistance at a future session (likely using a $5 \mathrm{~mm}$ camera to provide greater space and ease of movement). Session two indicated, endoscopic polypectomy with transabdominal, transcolonic assistance from the MiniLap device is feasible, although techniques need to be further refined.

Session three started with through a $12 \mathrm{~mm}$ umbilical port, a $12 \mathrm{~mm}$ right lowers quadrant port as well as inserting the MiniLap devices in the right and left upper quadrants as well as the pelvis for a self-retained bladder retractor. We used a MiniLap in the lower midline for the transabdominal transcolonic insertion, and then inserted a straight needle through the right lower quadrant. We subsequently inserted a U stitch around the area of purposed MiniLap insertion with the straight needle. The straight needle was then advanced back out the abdominal wall just next to the original insertion site. Using the stitch on both sides of the colon, the colon was tented up to allow for precise insertion of the MiniLap through the colon wall. However, the proximal side of the U stitch was placed too superficially and tore, creating a serosal (not complete) tear.

At this point, the MiniLap was still inserted transcolonically and the polypectomy was completed. The straight needle was backed into the abdominal cavity, a deeper bite was made for the proximal stitch, the needle was advanced back out the abdominal wall, the needle was removed, and the colotomy was closed with an intracorporeal knot. We then focused our attention to the use of an endostitch. With the use of the endostitch inserted very proximal to our camera port, we attempted to simulate the colotomy closure that would be performed in a single incision surgery. We were able to close the colotomy relatively easily; using the same techniques we had used during sessions $1 \& 2$ (Making the first throw and then the second while removing the MiniLap in an attempt to limit fecal contamination). We have actually used the MiniLap device for a transabdominal transcolonic polypectomy in our first patient.

This patient had a cecal polyp that we could visualize, but could not quite reach with our colonoscope even after full mobilization of the colon laparoscopically. We inserted the MiniLap device transcolonically, grasped nearby mucosa and presented the polyp to the snare of the endoscope. Unfortunately, the polyp had high grade dysplasia at the margins and the patient then underwent an ileocecectomy, but we plan to use the MiniLap in similar situations in the future to prevent the need for formal bowel resections. We also hope to develop more minimally invasive techniques (such as single incision) to assist in this endeavor.
As opposed to previous sessions when we obtained access through a $12 \mathrm{~mm}$ umbilical port, and $12 \mathrm{~mm}$ right lower quadrant port, in session four we instead inserted a single incision port in the umbilicus. We still inserted the MiniLap devices in the right and left upper quadrants as well as the pelvis for a selfretained bladder retractor. We also still used a MiniLap in the lower midline for the transabdominal transcolonic insertion. We inserted a $5 \mathrm{~mm}$, rigid, 30-degree camera as well as an endostitch through the single incision port. Using the MiniLap for grasping, we then created a U stitch around our area of planned colonic insertion. We did find that it was more difficult to place the stitch with precision, especially with the rigid camera in the way, but after some time, it was performed effectively.

We also did find that more torque was placed of the bowel when trying to place the suture all the way through the colon wall, although no serosal or transmural tears occurred. The polypectomy was performed with the use of the transcolonic MiniLap and the colonoscope without difficulty. The colotomy was once again closed by throwing two knots using the endostitch while removing the MiniLap at the same time to prevent fecal contamination. The excess suture was cut using endoshears and the closure appeared air tight. As mentioned previously, the $U$ stitches placed during this session did not seem as precise as previous sessions due to some compromised mobility. However, the closures were air tight and there was not significant narrowing seen intralumenally. In fact, we repeated this experiment several times in this session, with multiple colotomy closures, and even after all these attempts; we were still able to pass the colonoscope through these areas with relative ease.

We feel that we definitively demonstrated that transabdominal transcolonic polypectomies can be performed with a single incision port and some addition MiniLaps. In the future, we would like to refine this technique with the use of a $5 \mathrm{~mm}$ flexible tipped camera. During the last session, we attempted to use the techniques that we had developed to truly simulate a transabdominal, transcolonic polypectomy. We decided to use a two port technique with an umbilical port and a right lower quadrant port. We used a MiniLap in the low midline to be used as a self-retaining retractor for the bladder.

We used right upper and left upper MiniLaps for retraction and exposure, and a infraumbilical MiniLap in the midline for the polypectomy. The exposure was optimal. A U stitch was placed at the area of colotomy and the MiniLap was placed through the colon wall into the lumen. A simulated polypectomy was performed lifting mucosa from behind a fold and presenting it to the colonoscope. The colotomy was closed with the U stitch that had been previously placed. We ensured the closure was air tight and removed the instruments. We wanted this simulation to go as flawlessly as possible so we performed this with optimal exposure and did not attempt a single incision technique, although we had previously proved that this was feasible. 
We have successfully performed a transabdominal, transcolonic polypectomy with the use of the MiniLap and a colonoscope in one of our patients whose polyp was located behind a fold in the cecum. We plan to continue to use the MiniLap in similar situations to increase the number of patients eligible for colonoscopic polypectomies as well as decrease our procedural time in patients already undergoing colonoscopic polypectomies in the operating room.

\section{Acknowledgement}

Our research team would like to thank Leo Ondrovic, Ph.D. and Andrew Prather, M.D. for their help and intellectual support with this project.

\section{References}

1. Wolff WI, Shinya H (1975) Endoscopic polypectomy: therapeutic and clinicopathologic aspects. Cancer 36(2): 683-689.

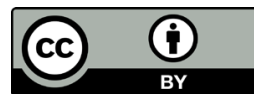

2. Fukami N, Lee JH (2006) Endoscopic treatment of large sessile and flat colorectal lesions. Curr Opin Gastroenterol 22(1): 54-59.

3. Church JM (2003) Experience in the endoscopic management of large colonic polyps. ANZ J Surg 73(12): 988-995.

4. Virk R, Gill S, Yoshida E, Radley S, Salh B (2010) Racial difference in the incidence of colorectal cancer. Can J Gastroenterol 24(1): 47-51.

5. Davis DM, Marcet JE, Frattini JC, Prather AD, Mateka JJ, et al. (2011) Is it time to lower the recommended screening age for colorectal cancer? J Am Coll Surg 213(3): 352-361.

6. Mandel JS (2008) Screening for colorectal cancer. Gastroenterol Clin North Am 37: 97-115.

7. Center MM, Jemal A, Ward E (2009) International Trends in Colorectal Cancer Incidence Rates. Cancer Epidemiol Biomarkers Prev 18(6): 1688-1694.

Your next submission with Juniper Publishers will reach you the below assets

- Quality Editorial service

- Swift Peer Review

- Reprints availability

- E-prints Service

- Manuscript Podcast for convenient understanding

- Global attainment for your research

- Manuscript accessibility in different formats ( Pdf, E-pub, Full Text, Audio)

- Unceasing customer service

Track the below URL for one-step submission https://juniperpublishers.com/online-submission.php 